\title{
Joint Phase Noise Estimation and Decoding in OFDM-IM
}

\author{
Qiaolin Shi ${ }^{1,2}$, Nan $\mathrm{Wu}^{2}$, Hua Wang ${ }^{2}$, Diep N. Nguyen ${ }^{1}$, Xiaojing Huang ${ }^{1}$ \\ ${ }^{1}$ Faculty of Engineering and Information Technology, University of Technology Sydney, NSW, Australia \\ ${ }^{2}$ School of Information and Electronics, Beijing Institute of Technology, Beijing, China \\ Email:Qiaolin.Shi@student.uts.edu.au,\{wunan,wanghua\}@bit.edu.cn, \{Diep.Nguyen,Xiaojing.Huang\}@uts.edu.au
}

\begin{abstract}
This paper proposes a low-complexity joint phase noise (PHN) estimation and decoding algorithm for orthogonal frequency division multiplexing relying on index modulation (OFDM-IM) systems. A factor graph (FG) is constructed based on the truncated discrete cosine transform (DCT) expansion model for the variation of PHN. In order to explicitly take into account the structured and sparse $a$ priori information of the frequency-domain symbols provided by the soft-in soft-out (SISO) decoder, the generalized approximate message passing (GAMP) algorithm is employed. Furthermore, to solve the unknown and nonlinear transform matrix problem introduced by the PHN, the mean-field (MF) method is invoked at the observation nodes on the FG. Monte Carlo simulations show the superiority of the proposed algorithm over the existing variational inference (VI) and extended Kalman filter (EKF) methods in terms of their bit error rate (BER) performance and complexity. In addition, we demonstrate that the OFDM-IM scheme outperforms its conventional OFDM counterpart in the presence of PHN.
\end{abstract}

Index Terms-Orthogonal frequency division multiplexing, index modulation, phase noise, discrete cosine transform, message passing receiver.

\section{INTRODUCTION}

Orthogonal frequency division multiplexing (OFDM) is one of the most popular multi-carrier techniques thanks to its effectiveness and low-complexity implementation in dispersive wideband channels. Recently, OFDM combined with index modulation (OFDM-IM) has emerged as a promising energyand spectral-efficient 5G technique [1]-[4]. In the OFDMIM scheme, some of the subcarriers are activated and their indices are used to transmit additional information on top of the conventional phase shift keying (PSK) or quadrature amplitude modulation (QAM) signal constellations. Hence, OFDM-IM is capable of providing a better tradeoff between the bandwidth efficiency and the bit error rate (BER) over its conventional OFDM counterpart [1]. Moreover, OFDMIM has the potential to attain a lower peak-to-average power ratio (PAPR) and to improve the energy efficiency compared to conventional OFDM under the same spectral efficiency [3] [4].

Nonetheless, similar to a conventional OFDM system, carrier frequency offset (CFO) error caused by Doppler shifts and phase noise (PHN) from local oscillators deteriorate the orthogonality among OFDM-IM subcarriers. Consequently, it

This work was supported by the National Science Foundation of China (NSFC) under Grant 61571041, Grant 61971041, and Grant 61471037. Corresponding author: Nan Wu. introduces intercarrier interference (ICI) and hence degrades the performance of OFDM-IM systems. However, most of OFDM-IM studies found in the literature assume perfectly known frequency-selective fading channels. In [5], null subcarriers were exploited to improve the accuracy of CFO estimation without accounting for the effect of PHN. In fact, in contrast to the channel impulse response (CIR) and the CFO, which can be assumed to be quasi-static compared to the OFDM-IM symbol rate, the PHN changes rapidly and thus cannot be mitigated using pilot symbols [6], [7]. As a result, it becomes much more challenging to design a low-complexity receiver for OFDM-IM with PHN impairment. Specifically, unlike the case without PHN, the maximum likelihood (ML) detector [1] performed within each subblock independently is not effective since the frequency-domain PSK/QAM symbols and the IM symbols from all subblocks are coupled together due to the existence of PHN. Thus the complexity of the optimal receiver becomes prohibitively high because an exhaustive search over all feasible combinations of PSK/QAM and IM symbols in a whole OFDM-IM block is required [1]. In addition, the PHN also distorts the estimation of PSK/QAM and IM symbols, especially when more than one subcarriers in a subblock are activated. Therefore, low-complexity joint PHN estimation and OFDM-IM detection algorithms are essential to enjoy advantages from the OFDM-IM scheme.

However, the existing joint PHN estimation and OFDM symbol detection problems are mainly discussed in uncoded scenarios [6]-[8]. In this article, we consider channel coding-aided systems and develop a low-complexity iterative decoding algorithm for OFDM-IM symbols communicating in frequency-selective fading channels affected by PHN. Recently, message passing algorithms based on factor graphs (FGs), such as belief propagation (BP) [9], mean-field (MF) approximation based variational methods [10] and generalized approximate message passing (GAMP) approach [11], have been widely used in the design of iterative wireless receivers. For spatial modulation aided systems, a GAMP equalizer is developed in [12] and further generalized in [13] and [14], where perfectly known block Rayleigh fading channels are considered.

To address the above issues, in this paper we propose a low-complexity message passing based joint PHN estimation and decoding method for OFDM-IM transmitting in dispersive channels. We take into account the sparse structure of the 


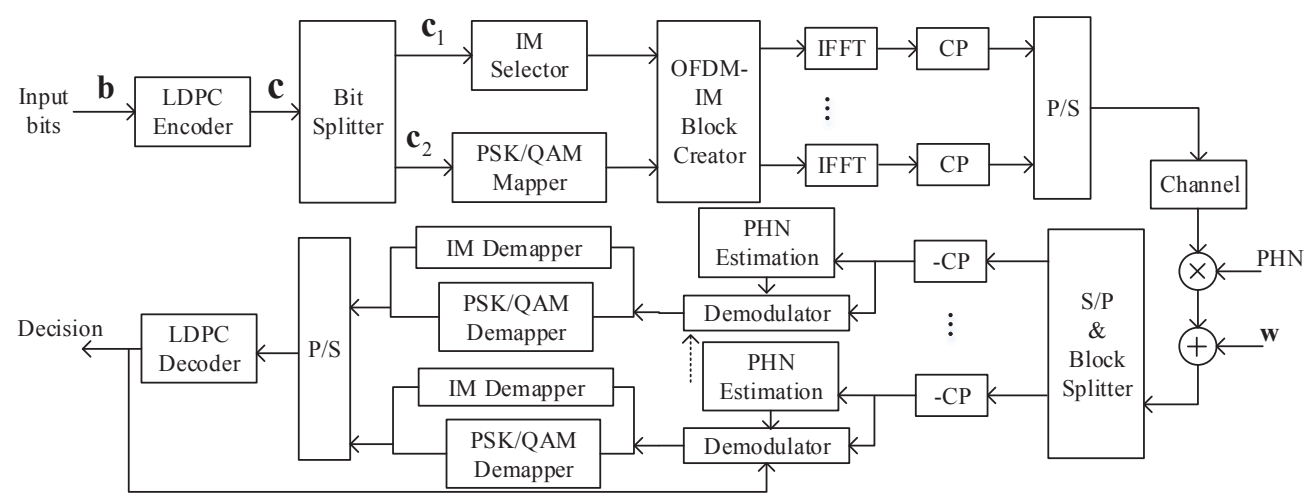

Fig. 1. The transceiver of the LDPC-coded OFDM-IM system.

frequency-domain OFDM-IM symbols and invoke the MF approach at the observation nodes to circumvent the nonlinear transform matrix problem imposed by the PHN. Instead of directly linearizing the PHN vector using the first-order Taylor expansion [6] or locally linearizing the system model using the previous PHN estimates [7], we employ the truncated discrete cosine transform (DCT) expansion model for approximating the PHN process ${ }^{1}$. In contrast to [15], where point estimation is made using the expectation-maximization algorithm, the measures of the accuracy of the DCT coefficients' estimates are calculated for providing the uncertainty over PHN estimates. Simulation results show that the proposed algorithm outperforms the conventional variational inference (VI) based and extended Kalman filter (EKF) based methods. Moreover, the OFDM-IM scheme with the proposed method is demonstrated to be more robust than its classical OFDM counterpart in the presence of PHN.

\section{SySTEM MODEL}

Consider $^{2}$ a turbo OFDM-IM system having $N_{q}$ subcarriers as depicted in Fig. 1, where the $N_{q}$ subcarriers are split into $G$ subblocks each containing $N_{g} \triangleq N_{q} / G$ subcarriers. In each subblock, only $K_{g}$ subcarriers are activated to transmit PSK/QAM symbols. Specifically, an $M_{i}$-length sequence of information bits $\mathbf{b}$ is encoded by a $R_{c}$-rate LDPC encoder, yielding an $M_{c}$-length codeword vector $\mathbf{c}$. These $M_{c}$ bits in c are then partitioned into two sequences, where the first sequence denoted as $\mathbf{c}_{1}$ is fed into the IM selector, whilst the second sequence denoted as $\mathbf{c}_{2}$ is mapped onto classic $M$-ary PSK/QAM constellation with alphabet $\mathcal{S} \triangleq\left\{s_{1}, \ldots, s_{M}\right\}$. As shown in Fig. 1, the bits in $\mathbf{c}_{1}$ are mapped into IM symbols

\footnotetext{
${ }^{1} \mathrm{~A}$ lowpass process such as the PHN process is well modeled by the DCT expansion, which has been widely used in image and audio compression areas.

${ }^{2}$ Notation: $X_{m n}$ and $x_{n}$ denote the $(m, n)$ th and the $n$th entry in matrix $\mathbf{X}$ and vector $\mathbf{x}$, respectively. $\mathbf{X}_{n,:}$ and $\mathbf{X}_{:, n}$ represent the $n$th row and the $n$th column of matrix $\mathbf{X}$, respectively. $\mathbf{I}_{N}$ indicates the $N \times N$ identity matrix. $\mathcal{D}(\mathbf{x})$ is a diagonal matrix with the elements of vector $\mathbf{x}$ on its diagonal. $\mathcal{N}_{c}\left(x ; \hat{m}_{x}, \hat{v}_{x}\right)$ represents the Gaussian random variable $x$ with the mean $\hat{m}_{x}$ and variance $\hat{v}_{x}$. The relationship $f(x)=c g(x)$ for some positive constant $c$ is written as $f(x) \propto g(x)$. We use $<\cdot>_{b(x)}$ to stand for the expectation with respect to a density $b(x) . \delta(\cdot)$ is the Dirac delta function and $\lfloor\cdot\rfloor$ is used to represent the integer floor operation. The operator $\odot$ denotes element-wise product and $\|\boldsymbol{\Omega}\|_{0}$ the size of set $\boldsymbol{\Omega} . \Re(\cdot)$ is the real part of a vector or scalar. The symbol $\mathbb{C}$ represents the field of complex.
}

according to a mapper $\mu_{1}:\{0,1\}^{M_{g 1}} \rightarrow \boldsymbol{\Omega}$, where $\boldsymbol{\Omega}=$ $\left\{\boldsymbol{\omega}_{1}, \ldots, \boldsymbol{\omega}_{\Upsilon}\right\}$ contains $\Upsilon=2^{M_{g 1}}$ legitimate combinations, each of which is formed with $K_{g}$ activated indices selected from $N_{g}$ available indices. In other words, the $\gamma$ th candidate of $\boldsymbol{\Omega}$ can be denoted by $\boldsymbol{\omega}_{\gamma}=\left[\omega_{\gamma, 1}, \ldots, \omega_{\gamma, N_{g}}\right]^{T} \in\{0,1\}^{N_{g}}$, where $\omega_{\gamma, i}=1$ if the $i$ th subcarrier is activated, and $\omega_{\gamma, i}=0$ otherwise. Therefore, we have $M_{g 1}=\log _{2} \Upsilon=\left\lfloor\log _{2}\left(\begin{array}{l}N_{g} \\ K_{g}\end{array}\right)\right\rfloor$ with $\left(\begin{array}{l}N_{g} \\ K_{g}\end{array}\right)$ representing the binomial coefficient. On the other hand, the bits in $\mathbf{c}_{2}$ are mapped onto classic PSK/QAM constellation according to a mapper $\mu_{2}:\{0,1\}^{M_{g 2}} \rightarrow \mathcal{S}$ and hence we have $M_{g 2}=\log _{2} M$.

By collecting the $K_{g}$ PSK/QAM symbols assigned to the $K_{g}$ active indices of the $g$ th subblock as $\mathbf{x}_{g, d}=$ $\left[x_{g, d}(1), \ldots, x_{g, d}\left(K_{g}\right)\right]^{T}, x_{g, d}\left(k_{g}\right) \in \mathcal{S}, g=1, \ldots, G$, we denote the frequency-domain symbols in the $g$ th subblock by $\mathbf{x}_{g}=\mathbf{I}_{g}^{0} \mathbf{x}_{g, d} \triangleq\left[x_{g}(1), \ldots, x_{g}\left(N_{g}\right)\right]^{T}$, where $\mathbf{I}_{g}^{0}$ consists of the $K_{g}$ columns from $\mathbf{I}_{N_{g}}$ having the activated indices. Here the activation pattern constraint is formulated as $\mathrm{x}_{g}^{\mathrm{AP}} \in \Omega$, where $x_{g}^{\mathrm{AP}}(n)=0$ if $x_{g}(n)=0$ and $x_{g}^{\mathrm{AP}}(n)=1$ otherwise. Next, we concatenate the $G$ symbol vectors using the OFDMIM block creator to form an OFDM-IM symbol, which can be written as $\mathbf{x}=\left[\mathbf{x}_{1}^{T}, \ldots, \mathbf{x}_{G}^{T}\right]^{T} \triangleq\left[x_{1}, \ldots, x_{N_{q}}\right]^{T} \in \mathbb{C}^{N_{q} \times 1}$, where the $n$th element in $\mathbf{x}$ obeys $x_{n} \in\{\mathcal{S}, 0\}$. Note that with a little 'abuse' of notation, we denote the bit vector corresponding to $\mathbf{x}_{g}$ by $\mathbf{c}_{g}=\left[c_{g, 1}^{\mathrm{IM}}, \ldots, c_{g, M_{g 1}}^{\mathrm{IM}}, c_{g, 1}^{\mathcal{S}}, \ldots, c_{g, M_{g 2}}^{\mathcal{S}}\right]^{T} \in$ $\{0,1\}^{M_{g}}$, where $c_{g, i}^{\mathrm{IM}}, i=1, \ldots, M_{g 1}$, is the $i$ th index selection bit, $c_{g, j}^{\mathcal{S}}, \jmath=1, \ldots, M_{g 2}$, the $\jmath^{\text {th }}$ symbol mapping bit, and $M_{g} \triangleq M_{g 1}+M_{g 2}$.

After employing the inverse fast Fourier transform (IFFT) and inserting an $L_{\mathrm{cp}}$-length cyclic prefix $(\mathrm{CP})$, the timedomain symbols are transmitted over an $L_{\mathrm{h}}$-tap $\left(L_{\mathrm{h}} \leqslant L_{\mathrm{cp}}\right)$ frequency-selective fading channel affected by PHN. At the receiver, the time-domain observations $\mathbf{r}=\left[r_{1}, \ldots, r_{N_{q}}\right]^{T}$ can be represented as

$$
\mathbf{r}=\frac{1}{\sqrt{K_{q}}} \mathcal{D}\left(e^{j \boldsymbol{\theta}}\right) \mathbf{F}^{H} \mathbf{H} \mathbf{x}+\mathbf{w},
$$

where $K_{q} \triangleq G K_{g}$ is used to normalize the transmitted power, $\boldsymbol{\theta}=\left[\theta_{1}, \ldots, \theta_{N_{q}}\right]^{T}$ is the discrete PHN sample vector, $\mathbf{F}$ is the discrete Fourier transform (DFT) matrix obeying $\mathbf{F}^{H} \mathbf{F}=$ 


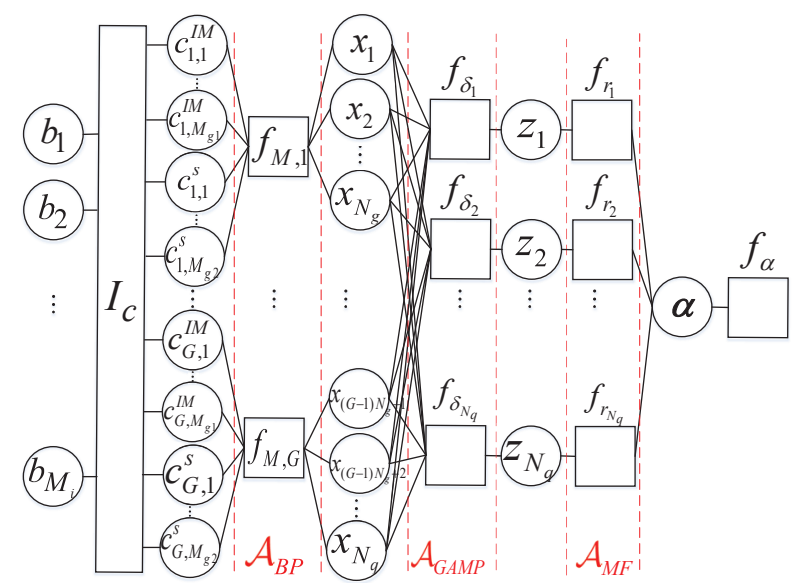

Fig. 2. Factor graph representation of the probabilistic model (3).

$N_{q} \mathbf{I}_{N_{q}}, \mathbf{H}=\mathcal{D}\left(\mathbf{F I}_{L_{\mathrm{h}}}^{0} \mathbf{h}\right)$ with $\mathbf{I}_{L_{\mathrm{h}}}^{0}$ being constructed by the first $L_{\mathrm{h}}$ columns from $\mathbf{I}_{N_{q}}$, and $\mathbf{w}$ is the zero-mean Gaussian noise vector with a variance of $2 \sigma^{2}$.

In order to reduce the total number of unknown PHN samples, we employ a truncated DCT expansion model [15] to interpret the PHN process, i.e., the vector $\mathbf{u} \triangleq e^{j \boldsymbol{\theta}}$ is approximated as $\mathbf{u}=\boldsymbol{\Phi} \boldsymbol{\alpha}$, where $\boldsymbol{\Phi} \in \mathbb{C}^{N_{q} \times L_{\mathrm{PN}}}$ is the known DCT matrix (see [15]), and $\boldsymbol{\alpha}=\left[\alpha_{1}, \ldots, \alpha_{L_{\mathrm{PN}}}\right]^{T}$ is the DCT coefficient vector to be estimated. To this end, the received vector can be rewritten as

$$
\mathbf{r}=\frac{1}{\sqrt{K_{q}}} \mathcal{D}(\boldsymbol{\Phi} \boldsymbol{\alpha}) \mathbf{F}^{H} \mathbf{H} \mathbf{x}+\mathbf{w} \triangleq \mathcal{D}(\mathbf{\Phi} \boldsymbol{\alpha}) \boldsymbol{\Lambda} \mathbf{x}+\mathbf{w},
$$

where the matrix $\boldsymbol{\Lambda}$ is defined as $\boldsymbol{\Lambda} \triangleq \frac{1}{\sqrt{K_{q}}} \mathbf{F}^{H} \mathbf{H}$. Based on (2), we obtain the joint a posteriori probability density function (pdf) of x and $\boldsymbol{\alpha}$

$p(\mathbf{x}, \boldsymbol{\alpha} \mid \mathbf{r}) \propto f_{M}(\mathbf{x}, \mathbf{c}, \mathbf{b}) P(\mathbf{x}) p(\boldsymbol{\alpha}) \prod_{n=1}^{N_{q}} f_{r_{n}}\left(z_{n}, \boldsymbol{\alpha}\right) f_{\delta_{n}}\left(z_{n}, \mathbf{x}\right)$,

where $f_{M}(\mathbf{x}, \mathbf{c}, \mathbf{b})$ represents the coding and modulation constraints; $P(\mathbf{x})$ and $p(\boldsymbol{\alpha})$ are the a priori information of the data symbol $\mathbf{x}$ and the DCT coefficient vector $\alpha$, respectively; $f_{r_{n}}\left(z_{n}, \boldsymbol{\alpha}\right) \triangleq p\left(r_{n} \mid z_{n}, \boldsymbol{\alpha}\right)=\mathcal{N}_{c}\left(r_{n} ;\left(\mathbf{\Phi}_{n,:} \boldsymbol{\alpha}\right) z_{n}, 2 \sigma^{2}\right)$ denotes the likelihood function with $z_{n} \triangleq \Lambda_{n,:}$. The factor graph corresponding to (3) is shown in Fig. 2.

\section{THE PROPOSED JOINT PHN ESTIMATION AND}

DECODING ALGORITHM IN THE OFDM-IM SCHEME

As seen in (2), the frequency-domain OFDM-IM symbols are coupled by the transform matrix $\mathcal{D}(\boldsymbol{\Phi} \boldsymbol{\alpha}) \mathbf{F}^{H} \mathbf{H}$, which imposes challenges on the detection. Moreover, the complexity of the optimal receiver of OFDM-IM symbols is much higher than that of traditional OFDM symbols, since it is required to detect both the subcarrier index-modulated and the PSK/QAM modulated symbols. In the following, we study a low-complexity method for joint PHN estimation and decoding in the OFDM-IM scheme. First, we observe that BP is compatible with the discrete probabilistic model, whilst MF has simple rules for updating the beliefs of continuous variables belonging to an exponential family [16], and GAMP can efficiently estimate a vector transformed from a known linear matrix [17]. We thus classify the factor nodes into three disjoint subsets, i.e., $\mathcal{A}_{\mathrm{BP}} \triangleq\left\{f_{M, g}\right\}_{g=1}^{G}, \mathcal{A}_{\mathrm{GAMP}} \triangleq\left\{f_{\delta_{n}}\right\}_{n=1}^{N_{q}}$, and $\mathcal{A}_{\mathrm{MF}} \triangleq\left\{f_{r_{n}}\right\}_{n=1}^{N_{q}}$. In this way, the messages can be easily calculated according to the amalgamated message passing rules.

Specifically, we use the extrinsic information provided by the soft-in soft-out (SISO) decoder, which is denoted by $\left\{P\left(c_{g, 1}^{\mathrm{IM}}\right), \ldots, P\left(c_{g, M_{g 1}}^{\mathrm{IM}}\right), P\left(c_{g, 1}^{\mathcal{S}}\right), \ldots, P\left(c_{g, M_{g 2}}^{\mathcal{S}}\right)\right\}$, to construct the sparse a priori information for the frequency-domain symbols in the $g$ th subblock, instead of approximating the prior probability mass function (pmf) to be in the separate form, i.e. $P(\mathbf{x})=\prod_{n=1}^{N_{q}} P\left(x_{n}\right)$. According to the mappers $\mu_{1}$ and $\mu_{2}$, we have

$$
\begin{gathered}
P\left(\mathbf{x}_{g}^{\mathrm{AP}}=\boldsymbol{\omega}_{\gamma}\right)=\prod_{i=1}^{M_{g 1}} P\left(c_{g, i}^{\mathrm{IM}}=c_{i}^{\boldsymbol{\omega}_{\gamma}}\right), \gamma=1, \ldots, \Upsilon, \\
P\left(x_{g, d}(k)=s_{m}\right)=\prod_{i=1}^{\log _{2} M} P\left(c_{g,(k-1) \log _{2} M+i}^{\mathcal{S}}=c_{i}^{s_{m}}\right), \\
m=1, \ldots, M, k=1, \ldots, K_{g},
\end{gathered}
$$

where the coded bit vectors corresponding to $\boldsymbol{\omega}_{\gamma}$ and $s_{m}$ are denoted by $\left[c_{1}^{\boldsymbol{\omega}_{\gamma}}, \ldots, c_{M_{g 1}}^{\boldsymbol{\omega}_{\gamma}}\right]^{T}$ and $\left[c_{1}^{s_{m}}, \ldots, c_{\log _{2} M}^{s_{m}}\right]^{T}$, respectively. Thus, the a priori pmf of $\mathrm{x}$ given by the SISO decoder can be obtained by

$$
\begin{aligned}
& P(\mathbf{x})= \prod_{g=1}^{G} P_{\mathbf{x}_{g}}\left(\mathbf{x}_{g}\right), \\
& P_{\mathbf{x}_{g}}\left(\mathbf{x}_{g}\right)=\sum_{\gamma=1}^{\Upsilon} P\left(\mathbf{x}_{g}^{\mathrm{AP}}=\boldsymbol{\omega}_{\gamma}\right)\left[\prod_{i \in \mathcal{I}^{\gamma}}\left(\sum_{s \in \mathcal{S}} \delta\left(x_{i}-s\right) P\left(x_{i}\right)\right)\right. \\
&\left.\cdot \prod_{\jmath \in \overline{\mathcal{I}}^{\gamma}} \delta\left(x_{\jmath}\right)\right],
\end{aligned}
$$

where $\mathcal{I}^{\gamma}$ is the support set of vector $\boldsymbol{\omega}_{\gamma}$, i.e., $\mathcal{I}^{\gamma}=\left\{i \mid \omega_{\gamma, i}=\right.$ $\left.1, i \in I_{g}\right\}$, with $I_{g} \triangleq\left\{(g-1) N_{g}+1: g N_{g}\right\}, \overline{\mathcal{I}}^{\gamma}$ is the complementary set of $\mathcal{I}^{\gamma}$, and $x_{i} \in\left\{x_{g, d}(1), \ldots, x_{g, d}\left(K_{g}\right)\right\}$.

Given (5) and the message from the variable node $x_{i}$ to the factor node $f_{M, g}$, i.e., $m_{x_{i} \rightarrow f_{M, g}}\left(x_{i}\right) \propto \mathcal{N}_{c}\left(x_{i} ; \hat{\vartheta}_{i}, v_{\vartheta_{i}}\right)$, the parameters of which are later updated by (18), the a posteriori pmf $b\left(x_{i}\right)$ of $x_{i}$ can be calculated by (6) for $x_{i}=s_{m}, s_{m} \in \mathcal{S}$, and by (7) for $x_{i}=0$. After some manipulations, we have

$$
b\left(x_{i}\right)= \begin{cases}\frac{D\left(x_{i}=s_{m}\right)}{\sum_{s \in \mathcal{S}} D\left(x_{i}=s\right)+E}, & x_{i} \in \mathcal{S}, \\ \frac{E}{\sum_{s \in \mathcal{S}} D\left(x_{i}=s\right)+E}, & x_{i}=0,\end{cases}
$$

where $D\left(x_{i}\right)$ and $E$ are given by (9) and (10), respectively. Then, the a posteriori mean and variance of $x_{i}$ can be calculated as

$$
\hat{x}_{i}=\sum_{s \in \mathcal{S}} s b\left(x_{i}=s\right), \quad v_{x_{i}}=\sum_{s \in \mathcal{S}}|s|^{2} b\left(x_{i}=s\right)-\left|\hat{x}_{i}\right|^{2} .
$$

Given the posterior pmf $b\left(x_{i}\right)$ in (8), the message from the factor node $f_{\delta_{n}}$ to the variable node $z_{n}$, i.e., $m_{f_{\delta_{n} \rightarrow z_{n}}}\left(z_{n}\right) \propto$ $\mathcal{N}_{c}\left(z_{n} ; \hat{\xi}_{n}, v_{\xi_{n}}\right)$, is calculated according to the GAMP rule 


$$
\begin{gathered}
b\left(x_{i}=s_{m}\right)=\sum_{\gamma: i \in \mathcal{I} \gamma} P\left(\mathbf{x}_{g}^{\mathrm{IM}}=\boldsymbol{\omega}_{\gamma}\right) \exp \left\{-\frac{\left|s_{m}-\hat{\vartheta}_{i}\right|^{2}}{v_{\vartheta_{i}}}\right\} P\left(x_{i}=s_{m}\right) \prod_{\jmath \in \mathcal{I} \gamma, \jmath \neq i}\left(\sum_{s \in \mathcal{S}} \exp \left\{-\frac{\left|s-\hat{\vartheta}_{\jmath}\right|^{2}}{v_{\vartheta_{\jmath}}}\right\} P\left(x_{\jmath}=s\right) \prod_{k \in \overline{\mathcal{I}}^{\gamma}} \exp \left\{-\frac{\left|\hat{\vartheta}_{k}\right|^{2}}{v_{\vartheta_{k}}}\right\} .\right. \\
b\left(x_{i}=0\right)=\exp \left\{-\frac{\left|\hat{\vartheta}_{i}\right|^{2}}{v_{\vartheta_{i}}}\right\} \cdot \sum_{\gamma: i \in \overline{\mathcal{I}}^{\gamma}} P\left(\mathbf{x}_{g}^{\mathrm{IM}}=\boldsymbol{\omega}_{\gamma}\right) \prod_{\jmath \in \overline{\mathcal{I}}^{\gamma}, \jmath \neq i} \exp \left\{-\frac{\left|\hat{\vartheta}_{\jmath}\right|^{2}}{v_{\vartheta_{\jmath}}}\right\} \prod_{k \in \mathcal{I}^{\gamma} \gamma}\left(\sum_{s \in \mathcal{S}} \exp \left\{-\frac{\left|s-\hat{\vartheta}_{k}\right|^{2}}{v_{\vartheta_{k}}}\right\} P\left(x_{k}=s\right)\right) . \\
D\left(x_{i}=s_{m}\right)=\sum_{\gamma: i \in \mathcal{I}^{\gamma}} P\left(\mathbf{x}_{g}^{\mathrm{IM}}=\boldsymbol{\omega}_{\gamma}\right) \exp \left\{-\frac{\left|s_{m}\right|^{2}-2 \Re\left\{s_{m} \hat{\vartheta}_{i}^{*}\right\}}{v_{\vartheta_{i}}}\right\} P\left(x_{i}=s_{m}\right) \prod_{\jmath \in \mathcal{I}^{\gamma}, \jmath \neq i}\left(\sum_{s \in \mathcal{S}} \exp \left\{-\frac{|s|^{2}-2 \Re\left\{s \hat{\vartheta}_{\jmath}^{*}\right\}}{v_{\vartheta_{\jmath}}}\right\} P\left(x_{\jmath}=s\right)\right) . \\
E=\sum_{\gamma: i \in \overline{\mathcal{I}} \gamma} P\left(\mathbf{x}_{g}^{\mathrm{IM}}=\boldsymbol{\omega}_{\gamma}\right) \prod_{\jmath \in \mathcal{I}^{\gamma}}\left(\sum_{s \in \mathcal{S}} \exp \left\{-\frac{|s|^{2}-2 \Re\left\{s \hat{\vartheta}_{\jmath}^{*}\right\}}{v_{\vartheta_{\jmath}}}\right\} P\left(x_{\jmath}=s\right)\right) .
\end{gathered}
$$

([11, Eqs. (2a) and (2b)]) by

$$
v_{\xi_{n}}=\sum_{i}\left|\Lambda_{n, i}\right|^{2} v_{x_{i}}, \quad \hat{\xi}_{n}=\sum_{i} \Lambda_{n, i} \hat{x}_{i}-\hat{\zeta}_{n} v_{\xi_{n}},
$$

where $\hat{\zeta}_{n}$ is related to the estimate of $z_{n}$ and is later updated by (17). Then given the posterior pdf $b(\boldsymbol{\alpha}) \propto \mathcal{N}\left(\boldsymbol{\alpha} ; \hat{\boldsymbol{\alpha}}, \mathbf{V}_{\boldsymbol{\alpha}}\right)$, which is later updated by (19), the message $m_{f_{r_{n} \rightarrow z_{n}}}\left(z_{n}\right)$ is calculated by using the MF method [10, Eq. (18)]

$$
\begin{aligned}
m_{f_{r_{n} \rightarrow z_{n}}}\left(z_{n}\right) & \propto \exp \left\{<\log f_{r_{n}}\left(z_{n}, \boldsymbol{\alpha}\right)>_{b(\boldsymbol{\alpha})}\right\} \\
& \approx \mathcal{N}_{c}\left(z_{n} ; \hat{\eta}_{n}, v_{\eta_{n}}\right),
\end{aligned}
$$

where

$$
\hat{\eta}_{n}=\frac{r_{n}}{\hat{\varrho}_{u_{n}}}(\mathbf{\Phi} \hat{\boldsymbol{\alpha}})_{n}^{*}, \quad v_{\eta_{n}}=2 \sigma^{2} / \varrho_{u_{n}}
$$

with

$$
\begin{array}{r}
\hat{\varrho}_{u_{n}}=\sum_{i=1}^{L_{\mathrm{PN}}}\left|\Phi_{n, i}\right|^{2}\left(V_{i, i}+\left|\hat{\alpha}_{i}\right|^{2}\right)+\sum_{i=1}^{L_{\mathrm{PN}}} \sum_{\jmath=1, \jmath>i}^{L_{\mathrm{PN}}} \Phi_{n, i} \Phi_{n, \jmath} \\
\cdot 2 \Re\left(V_{i, \jmath}+\hat{\alpha}_{i}^{*} \hat{\alpha}_{\jmath}\right) .
\end{array}
$$

In (15), $\hat{\alpha}_{i}$ and $V_{i, i}$ are the $i$ th and the $(i, i)$ th entries of $\hat{\boldsymbol{\alpha}}$ and $\mathbf{V}_{\boldsymbol{\alpha}}$, respectively, which are later updated by (19). Given the messages $m_{f_{\delta_{n} \rightarrow z_{n}}}\left(z_{n}\right)$ and $m_{f_{r_{n} \rightarrow z_{n}}}\left(z_{n}\right)$, the a posteriori pdf of $z_{n}$ can be formulated as $b\left(z_{n}\right) \propto \mathcal{N}_{c}\left(z_{n} ; \hat{z}_{n}, v_{z_{n}}\right)$, where

$$
v_{z_{n}}=\left(v_{\xi_{n}}^{-1}+v_{\eta_{n}}^{-1}\right)^{-1}, \quad \hat{z}_{n}=v_{z_{n}}\left(\hat{\xi}_{n} v_{\xi_{n}}^{-1}+\hat{\eta}_{n} v_{\eta_{n}}^{-1}\right) .
$$

Then the output scalar function $g_{\text {out }}\left(\hat{\xi}_{n}, v_{\xi_{n}}, \hat{\eta}_{n}, v_{\eta_{n}}\right)$ defined in original GAMP is updated by ([11, Eqs. (3a) and (3b)])

$$
\begin{gathered}
\hat{\zeta}_{n}=g_{\text {out }}\left(\hat{\xi}_{n}, v_{\xi_{n}}, \hat{\eta}_{n}, v_{\eta_{n}}\right)=\frac{\hat{z}_{n}-\hat{\xi}_{n}}{v_{\xi_{n}}}=\frac{\hat{\eta}_{n}-\hat{\xi}_{n}}{v_{\eta_{n}}+v_{\xi_{n}}}, \\
v_{\zeta_{n}}=-\frac{\partial}{\partial \hat{\xi}_{n}} g_{\text {out }}\left(\hat{\xi}_{n}, v_{\xi_{n}}, \hat{\eta}_{n}, v_{\eta_{n}}\right)=\frac{1}{v_{\xi_{n}}}\left(1-\frac{v_{z_{n}}}{v_{\xi_{n}}}\right) .
\end{gathered}
$$

In the GAMP algorithm ([11, Eqs. (4a) and (4b)]), the variance and the mean of the message $m_{x_{i} \rightarrow f_{M, g}}\left(x_{i}\right) \propto \mathcal{N}_{c}\left(x_{i} ; \hat{\vartheta}_{i}, v_{\vartheta_{i}}\right)$ are calculated by

$$
v_{\vartheta_{i}}=\left(\sum_{n}\left|\Lambda_{n, i}\right|^{2} v_{\zeta_{n}}\right)^{-1}, \hat{\vartheta}_{i}=v_{\vartheta_{i}} \sum_{n} \hat{\zeta}_{n} \Lambda_{n, i}^{*}+\hat{x}_{i} .
$$

Then, given the a posteriori information of the additional variable $z_{n}$, the message passing from the observation node $r_{n}$ to the DCT coefficient vector $\boldsymbol{\alpha}$ can be updated as

$$
\begin{aligned}
& m_{f_{r_{n}} \rightarrow \boldsymbol{\alpha}}(\boldsymbol{\alpha}) \propto \exp \left\{<\log f_{r_{n}\left(\boldsymbol{\alpha}, z_{n}\right)}>_{b\left(z_{n}\right)}\right\} \\
\propto & \exp \left\{\frac{2 \Re\left\{r_{n}^{*} \hat{z}_{n}(\boldsymbol{\Phi} \boldsymbol{\alpha})_{n}\right\}-\left|(\boldsymbol{\Phi} \boldsymbol{\alpha})_{n}\right|^{2}\left(\left|\hat{z}_{n}\right|^{2}+v_{z_{n}}\right)}{2 \sigma^{2}}\right\} .
\end{aligned}
$$

Given the message $m_{f_{r_{n}} \rightarrow \boldsymbol{\alpha}}(\boldsymbol{\alpha})$, the posterior pdf of the DCT coefficient vector is calculated by ([9, Eqs. (5) and (6)])

$$
\begin{aligned}
b(\boldsymbol{\alpha}) & \propto p(\boldsymbol{\alpha}) \prod_{n=1}^{N_{q}} m_{f_{r_{n}} \rightarrow \boldsymbol{\alpha}}(\boldsymbol{\alpha}) \\
& \propto \exp \left\{\frac{2 \Re\left\{\left(\mathbf{r} \odot \hat{\mathbf{z}}^{*}\right)^{H} \boldsymbol{\Phi} \boldsymbol{\alpha}\right\}-\boldsymbol{\alpha}^{H} \boldsymbol{\Phi}^{H} \mathcal{D}\left(\hat{\varrho}_{z}\right) \boldsymbol{\Phi} \boldsymbol{\alpha}}{2 \sigma^{2}}\right\},
\end{aligned}
$$

where $\hat{\varrho}_{z}=\left[\left|\hat{z}_{1}\right|^{2}+v_{z_{1}}, \ldots,\left|\hat{z}_{N_{q}}\right|^{2}+v_{z_{N_{q}}}\right]^{T}$, while its covariance matrix and mean vector are

$$
\mathbf{V}_{\boldsymbol{\alpha}}=2 \sigma^{2}\left(\boldsymbol{\Phi}^{H} \mathcal{D}\left(\hat{\boldsymbol{\varrho}}_{z}\right) \boldsymbol{\Phi}\right)^{-1}, \hat{\boldsymbol{\alpha}}=\frac{1}{2 \sigma^{2}} \mathbf{V}_{\boldsymbol{\alpha}} \boldsymbol{\Phi}^{H}\left(\mathbf{r} \odot \hat{\mathbf{z}}^{*}\right) .
$$

Finally, the extrinsic log-likelihood ratios (LLRs) of the subcarrier selection bits and the PSK/QAM mapping bits can be calculated based on the Turbo principle [13], [14].

We summarize the proposed DCT model based joint PHN estimation and decoding algorithm for OFDM-IM symbols in Algorithm 1.

\section{A. Complexity Analysis}

In Table 1, we compare the complexity of the proposed algorithm with the existing approaches. Considering that all algorithms employ the standard Bahl, Cocke, Jelinek, and Raviv (BCJR) decoding, we only focus on the complexity of the iteration between the demodulator and the PHN estimator. For the proposed method, the demodulator requires $\mathcal{O}\left(G N_{g} M\right)$ operations for the calculations of (9) and (10), and two $N_{q}$-point FFTs for calculating $\left\{\hat{\xi}_{n}\right\}_{n=1}^{N_{q}}$ and $\left\{\hat{\vartheta}_{i}\right\}_{i=1}^{N_{q}}$ which are in the order of $\mathcal{O}\left(2 N_{q} \log N_{q}\right)$ [17]. For the optimal receiver, a complexity order of $\mathcal{O}\left(2^{G M_{g}}\right)$ is required for the soft demodulation. Furthermore, the existing PHN estimation methods in [6] and [7] are combined with the proposed soft demodulation algorithm. The VI approach in [6] has to calculate an inversion of a nontrivial matrix which requires a complex order of $\mathcal{O}\left(N_{q}^{3}\right)$. The complexity of the EKF method in [7] is in the order of $\mathcal{O}\left(N_{q}\right)$ by using the linearized system model. For the proposed DCT model based PHN estimation 
TABLE I

COMPARISONS OF COMPUTATIONAL COMPLEXITY (FOR EXAMPLE: $N_{q}=256, L_{P N}=2, G=64, M=4$, AND $M_{g}=4$.)

\begin{tabular}{|l|r|r|r|r|}
\hline Algorithm & Complexity of the demodulator & Value & Complexity of the PHN estimator & $\mathcal{O}\left(2 N_{q} L_{P N}\right)$ \\
\hline Proposed & $\mathcal{O}\left(G N_{g} M+2 N_{q} \log N_{q}\right)$ & $\mathcal{O}(5120)$ & $\mathcal{O}(1024)$ \\
\hline Optimal Detector & $\mathcal{O}\left(2^{G M_{g}}\right)$ & $\mathcal{O}\left(1.2 \times 10^{77}\right)$ & - & - \\
\hline EKF [7] & - & - & $\mathcal{O}\left(N_{q}\right)$ & $\mathcal{O}(256)$ \\
\hline VI [6] & - & - & $\mathcal{O}\left(N_{q}^{3}\right)$ & $\mathcal{O}\left(1.7 \times 10^{7}\right)$ \\
\hline
\end{tabular}

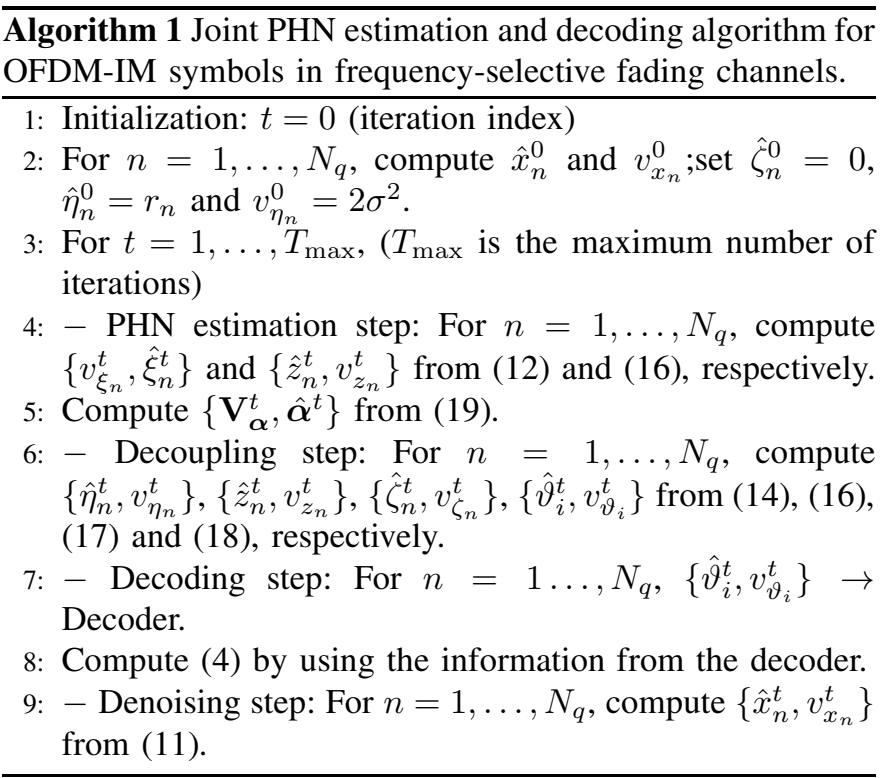

algorithm, $\mathcal{O}\left(2 N_{q} L_{\mathrm{PN}}\right)$ operations are needed for calculating (14) and (19) assuming $\mathcal{D}\left(\hat{\varrho}_{z}\right) \approx \hat{\varrho}_{z} \mathbf{I}_{N_{q}}$, where $\hat{\varrho}_{z}$ is the average of the elements in $\hat{\varrho}_{z}$.

\section{Simulation Results}

In this section, the performance of the proposed joint PHN estimation and decoding algorithm for OFDM-IM symbols is evaluated and compared with that of conventional VI [6] and EKF [7] approaches. In all simulations, we employ a 0.73-rate LDPC code with a codeword length of 6048 . The number of available subcarriers in an OFDM-IM block is selected to be 256 , i.e. $N_{q}=256$. A Rayleigh fading channel [1], the CIRs of which are generated according to the distributions $\mathcal{N}\left(h_{l} ; 0,0.1\right), l=1, \ldots, 10$, is considered. The length of the $\mathrm{CP}$ is set to be 16 . The Wiener model, i.e., $\theta_{n}=\theta_{n-1}+\triangle_{n}$ with $\triangle_{n}$ being a Gaussian random variable with zero mean and standard deviation $\sigma_{\triangle}$, is used to generate the PHN samples [15]. One pilot symbol is inserted every 64 subcarriers to make the algorithm bootstrap. The maximum number of iterations between the demodulator and the decoder is $T_{\max }=20$. According to the design guide given in [18] that the OFDM-IM scheme explicitly outperforms its OFDM counterpart when the throughput is relatively low, we only consider low-order modulations such as binary phase shift keying (BPSK) and quadrature phase shift keying (QPSK).

Fig. 3 shows the BER performance of the proposed algorithm for the OFDM-IM characterized by $\left\{M=2, N_{g}=\right.$ $\left.4, K_{g}=1\right\}$ (denoted by "Proposed") and for traditional BPSK modulated OFDM (denoted by "OFDM BPSK"). The Wiener models with standard deviations $\sigma_{\triangle}=0.5^{\circ}$ and $3^{\circ}$

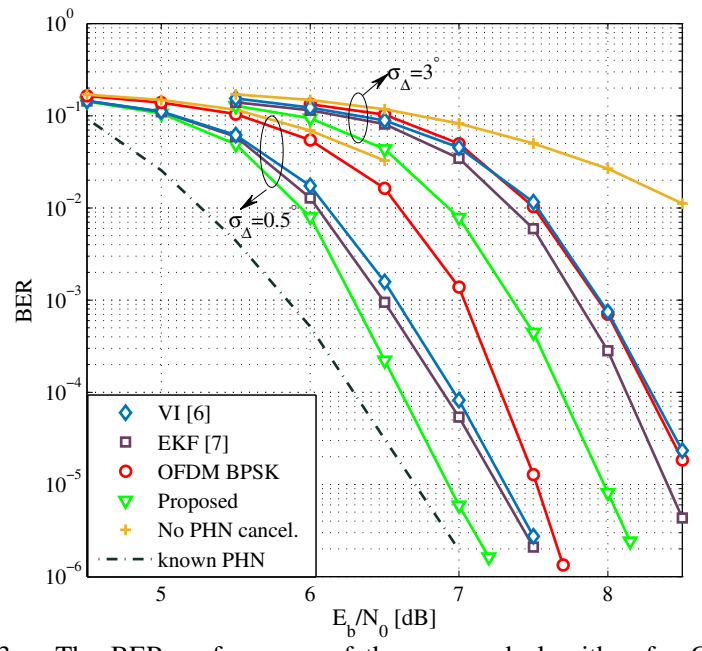

Fig. 3. The BER performance of the proposed algorithm for OFDM-IM $(2,4,1)$ and classic BPSK-modulated OFDM compared with existing VI [6] and EKF [7] methods. Wiener PHN models with standard deviations of $\sigma_{\triangle}=$ $0.5^{\circ}$ and $3^{\circ}$ are considered.

are both considered. Additionally, the existing VI [6] and EKF [7] methods proposed for joint PHN estimation and uncoded OFDM detection are now extended to the OFDMIM scheme by combining them with our proposed message passing based demodulating algorithm. For comparison, the BER curve without PHN mitigation (denoted by "No PHN cancel.") is provided. The BER curve of the OFDM-IM system with perfectly known PHN (denoted by "known PHN") is also plotted as a benchmark. We observe that the performance loss of the proposed method compared with the case with known PHN is negligible when $\sigma_{\triangle}=0.5^{\circ}$, which demonstrates the effectiveness of the DCT model based PHN mitigation algorithm. As expected, significant performance loss is seen if we ignore the PHN impairment. It is also seen that the proposed method outperforms the VI and EKF approaches especially for severe PHN. The VI method delivers poor BER performance because it directly linearizes the system model by approximating the vector of PHN samples based on the firstorder Taylor expansion. The accuracy of this approximation degrades with the increase of the PHN intensity. For the EKF, the system model is locally linearized using the first-order Taylor expansion given the PHN estimates obtained from the previous iteration, which introduces significant performance loss for strong PHN. Moreover, compared to classic OFDM, the OFDM-IM system employing the proposed method is more robust to the effect of PHN.

In Fig. 4, we compare the BER performance of the OFDMIM characterized by $\left\{M=4, N_{g}=4, K_{g}=3\right\}$ (denoted by "Proposed") with conventional QPSK modulated OFDM (denoted by "OFDM QPSK") having the same spectral efficiency. 


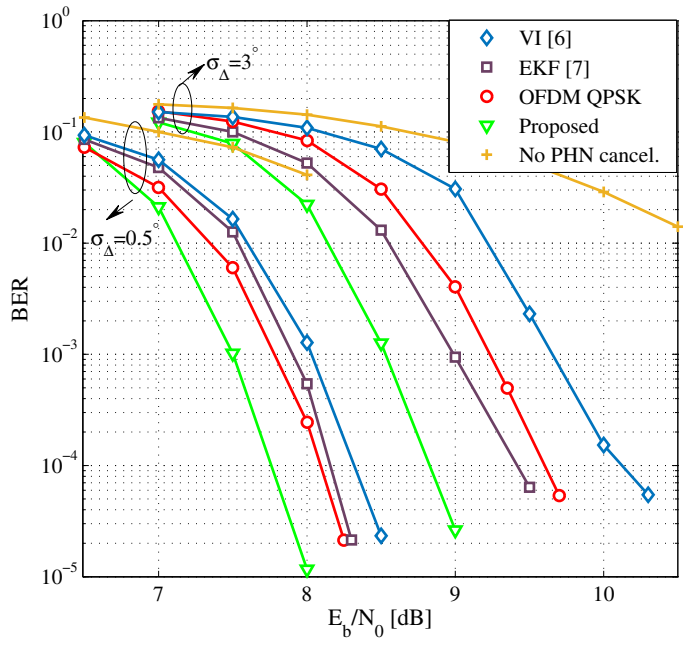

Fig. 4. The BER performance of the proposed algorithm for OFDM-IM $(4,4,3)$ and classic QPSK-modulated OFDM compared with existing VI [6] and EKF [7] methods. Wiener PHN models with standard deviations of $\sigma_{\triangle}=$ $0.5^{\circ}$ and $3^{\circ}$ are considered.

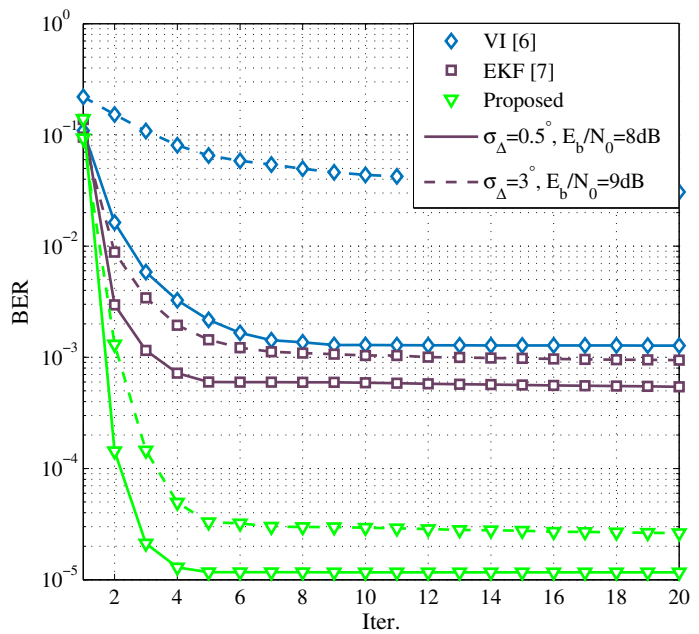

Fig. 5. The BER performance of the proposed algorithm for OFDM-IM $(4,4,3)$ and classic QPSK-modulated OFDM compared with existing VI [6] and EKF [7] methods in terms of BER versus iteration index. Wiener PHN models with standard deviations of $\sigma_{\triangle}=0.5^{\circ}$ and $3^{\circ}$ are considered.

The BER performance of three aforementioned algorithms all degrades upon increasing the standard deviation of the Wiener model. Similar trend to that in Fig. 3 is observed that the proposed algorithm exhibits the best performance among them. Fig. 5 shows the BER performance as a function of the number of iterations under the same conditions of Fig. 4. We observe that the convergence of the VI method is slow compared to the EKF and the proposed algorithm, which explains its error floor in Fig. 4 when $\sigma_{\triangle}=3^{\circ}$.

\section{CONCLUSions}

In this paper, we studied a low-complexity joint PHN estimation and OFDM-IM detection in LDPC-coded systems. The PHN process was approximated by the DCT model. Based on this we explicitly represented the sparse a priori information and constructed a proper FG according to the joint $a$ posteriori pdf. The GAMP technique defined over this FG was modified for the soft demodulation. Then the MF algorithm was applied at the observation nodes to calculate the extrinsic information for the frequency-domain OFDMIM symbols and update the PHN estimates in a Turbo fashion. Our simulation results showed that the proposed algorithm: 1) significantly decreased the complexity of the optimal receiver; and 2) outperformed the existing VI and EKF approaches with acceptable complexity. Also, we observed that the OFDM-IM scheme employing the proposed method is more robust than its classic OFDM counterpart.

\section{REFERENCES}

[1] E. Basar, U. Aygolu, E. Panayirci, and H. V. Poor, "Orthogonal frequency division multiplexing with index modulation," IEEE Trans. Signal Process., vol. 61, no. 22, pp. 5536-5549, Nov 2013.

[2] M. Wen, B. Ye, E. Basar, Q. Li, and F. Ji, "Enhanced orthogonal frequency division multiplexing with index modulation," IEEE Trans. Wireless Commun., vol. 16, no. 7, pp. 4786-4801, July 2017.

[3] D. Tsonev, S. Sinanovic, and H. Haas, "Enhanced subcarrier index modulation (SIM) OFDM," in Proc. IEEE GLOBECOM Workshops, pp. 728-732, Dec 2011.

[4] L. Zhao, H. Zhao, K. Zheng, and Y. Yang, "A high energy efficient scheme with selecting sub-carriers modulation in OFDM system," in Proc. IEEE ICC, June 2012, pp. 5711-5715.

[5] Z. Yang, F. Chen, B. Zheng, M. Wen, and W. Yu, "Carrier frequency offset estimation for OFDM with generalized index modulation systems using inactive data tones," IEEE Commun. Lett., vol. 22, no. 11, pp. 2302-2305, Nov 2018

[6] D. D. Lin and T. J. Lim, "The variational inference approach to joint data detection and phase noise estimation in OFDM," IEEE Trans. Signal Process., vol. 55, no. 5, pp. 1862-1874, May. 2007.

[7] O. H. Salim, A. A. Nasir, H. Mehrpouyan, W. Xiang, S. Durrani, and R. A. Kennedy, "Channel, phase noise, and frequency offset in OFDM systems: Joint estimation, data detection, and hybrid cramer-rao lower bound," IEEE Trans. Commun., vol. 62, no. 9, pp. 3311-3325, Sep. 2014.

[8] R. Wang, H. Mehrpouyan, M. Tao, and Y. Hua, "Channel estimation, carrier recovery, and data detection in the presence of phase noise in OFDM relay systems," IEEE Trans. Wireless Commun., vol. 15, no. 2, pp. 1186-1205, Feb 2016.

[9] F. R. Kschischang, B. J. Frey, and H. A. Loeliger, "Factor graphs and the sum-product algorithm," IEEE Trans. Inform. Theory, vol. 47, no. 2, pp. 498-519, Feb. 2001.

[10] J. Winn and C. M. Bishop, "Variational message passing," Journal of Machine Learning Research, vol. 6, no. Apr, pp. 661-694, 2005.

[11] S. Rangan, "Generalized approximate message passing for estimation with random linear mixing," in Proc. IEEE Inf. Symp. Inf. Theory. IEEE, 2011, pp. 2168-2172.

[12] Y. Zhao, Y. Xiao, P. Yang, B. Dong, R. Shi, and K. Deng, "Generalized approximate message passing aided frequency domain turbo equalizer for single-carrier spatial modulation," IEEE Trans. Veh. Technol., vol. 67, no. 4, pp. 3630-3634, April 2018.

[13] X. Meng, S. Wu, L. Kuang, D. Huang, and J. Lu, "Multi-user detection for spatial modulation via structured approximate message passing," IEEE Commun. Lett., vol. 20, no. 8, pp. 1527-1530, Aug 2016.

[14] L. Wei, J. Zheng, and Q. Liu, "Approximate message passing detector for index modulation with multiple active resources," IEEE Trans. Veh. Technol., vol. 68, no. 1, pp. 972-976, Jan 2019.

[15] N. Noels, J. Bhatti, H. Bruneel, and M. Moeneclaey, "Block-processing soft-input soft-output demodulator for coded PSK using DCT-based phase noise estimation," IEEE Trans. Commun., vol. 62, no. 8, pp. 2939 2950, Aug. 2014

[16] E. Riegler, G. E. Kirkelund, C. N. Manchon, M. Badiu, and B. H. Fleury, "Merging belief propagation and the mean field approximation: A free energy approach," IEEE Trans. Inf. Theory, vol. 59, no. 1, pp. 588-602, Jan. 2013.

[17] Q. Shi, N. Wu, X. Ma, and H. Wang, "Frequency-domain joint channel estimation and decoding for faster-than-nyquist signaling," IEEE Trans. Commun., vol. 66, no. 2, pp. 781-795, Feb. 2018.

[18] N. Ishikawa, S. Sugiura, and L. Hanzo, "Subcarrier-index modulation aided OFDM - will it work?” IEEE Access, vol. 4, pp. 2580-2593, 2016. 